\title{
Weakly anisotropic frustrated zigzag spin chain
}

\author{
D.V.Dmitriev and V.Ya.Krivnov \\ Joint Institute of Chemical Physics of RAS, Kosygin str.4, 119334, Moscow, Russia.
}

(Dated:)

\begin{abstract}
The frustrated spin-1/2 model with weakly anisotropic ferromagnetic nearest-neighbor and antiferromagnetic next-nearest-neighbor exchanges is studied with use of variational mean-field approach, scaling estimates of the infrared divergencies in the perturbation theory and finite-size calculations. The ground state phase diagram of this model contains three phases: the ferromagnetic phase, the commensurate spin-liquid phase and the incommensurate phase. The non-trivial behavior of the boundaries between these phases and the character of the phase transitions in case of weak anisotropy are determined.
\end{abstract}

\section{INTRODUCTION}

The quantum spin chains with nearest-neighbor (NN) $J_{1}$ and next-nearest-neighbor (NNN) interactions $J_{2}$ have been a subject of numerous studies [1]. The model with both antiferromagnetic interactions $J_{1}, J_{2}>0$ (AF-AF model) is well studied [2, 3, 4, 5, 6, 7]. Lately, there has been considerable interest in the study of F-AF model with the ferromagnetic NN and the antiferromagnetic NNN interactions $\left(J_{1}<0, J_{2}>0\right)[8,19,10,11,12$, 13, 14]. One of the reasons is understanding of intriguing magnetic properties of a novel class of edge-sharing copper oxides which are described by the F-AF model [15, 16, 17, 18, 19, 20]. In particular, these copper oxides show at low temperature a tendency to the formation of the incommensurate state with helicoidal magnetic ordering.

The Hamiltonian of the F-AF model is

$$
H=-\sum_{n=1}^{N}\left(S_{n}^{x} S_{n+1}^{x}+S_{n}^{y} S_{n+1}^{y}+\Delta_{1} S_{n}^{z} S_{n+1}^{z}\right)+J \sum_{n=1}^{N}\left(S_{n}^{x} S_{n+2}^{x}+S_{n}^{y} S_{n+2}^{y}+\Delta_{2} S_{n}^{z} S_{n+1}^{z}\right)
$$

where we put $J_{1}=-1$ and $J_{2}=J>0$ and the periodic boundary conditions are implied.

The isotropic case of this model $\left(\Delta_{1}=\Delta_{2}=1\right)$ is intensively studied last years [11, 12, 21, 22, 23]. It is known that the ground state of the isotropic version of the model (11) is ferromagnetic at $0<J<1 / 4$ and it becomes a singlet incommensurate state for $J>1 / 4[24,25]$. The phase transition at $J=1 / 4$ is the second order one.

The model with the anisotropy of exchange interactions is less studied, especially for the case of the small anisotropy. For example, the phase diagram of the model (1) with $\Delta_{1}=\Delta_{2}$ has been studied in Ref. [26] using the method of level spectroscopy. Unfortunately, this method becomes unreliable for $J \approx 1 / 4$ and $\Delta_{1}=\Delta_{2} \approx 1$ because of strong finite-size effects.

In real chain compounds the exchange interactions are anisotropic. The microscopic origin of these interactions is the spin-orbit coupling. The indication on the anisotropy is a dependence of the saturation field on the direction of the external magnetic field [16]. Though, as a rule, the anisotropy is weak (for example, for edge-shared cuprate $\mathrm{LiCuVO}_{4}$ ESR detected a $6 \%$ anisotropy [27]), it can change the transition point from commensurate to incommensurate states as well as the behavior of the model (1) in the vicinity of the transition point. Besides, the frustration parameter $\left|J_{2} / J_{1}\right|=J$ estimated for some edge-sharing copper oxides is close to the quantum critical point 1/4 (for example, $J \sim 0.28-0.3$ for compound $\mathrm{Li}_{2} \mathrm{ZrCuO}_{4}[28]$ ). Therefore, taking into account both the frustration effects and the small exchange anisotropy near the transition point can be important for the analysis of the experimental data related to these compounds.

In the isotropic case of (1) the ferromagnetic state is $(N+1)$-fold degenerated at $0<J<1 / 4$. Weak easy-plane anisotropy $\Delta_{1}, \Delta_{2}<1$ lifts this degeneracy and the ground state is in the sector with total $S^{z}=0$ at small $J$. One can expect that the increase of $J$ induces the phase transition at some $J_{c}$ to the incommensurate phase with $S^{z}=0$. Besides, the character of this transition can be different from that in the isotropic case.

In our analysis we focus on the behavior of the F-AF model (11) near the transition point from the commensurate to the incommensurate ground state and the influence of the weak anisotropic interaction on the $T=0$ phase diagram.

*Electronic address: dmitriev@deom.chph.ras.ru 
For simplicity we concentrate our attention on the particular case of the Hamiltonian (11) with $\Delta_{2}=1$

$$
H=-\sum\left(S_{n}^{x} S_{n+1}^{x}+S_{n}^{y} S_{n+1}^{y}+\Delta S_{n}^{z} S_{n+1}^{z}-\frac{1}{4}\right)+J \sum\left(\mathbf{S}_{n} \cdot \mathbf{S}_{n+2}-\frac{1}{4}\right)
$$

(We added here constants for convenience.)

However, we will show that the results for the model (1) with both $\Delta_{1} \neq 1$ and $\Delta_{2} \neq 1$ are qualitatively similar to those for the model (2).

The paper is organized as follows. In Sec.II we consider a qualitative physical picture of the ground state phase diagram of the model (2) based on the classical approximation. In Sec.III we study the phase diagram of the model (2) using the variational mean-field approach. The scaling estimates of the perturbation theory for the easy-plane case of the model (2) at $J<1 / 4$ are presented in Sec.IV. In Sec.V we estimate infrared divergencies in the perturbation theory near the transition point $J=1 / 4$. Sec.VI is devoted to the phase transition in the easy-axis case of the model (2). In Sec.VII we present the phase diagram of the model (11) in the case $\Delta_{1}=\Delta_{2}$ and summarize our results.

\section{CLASSICAL APPROXIMATION}

Let us start from the classical picture of the ground state of the model (2). In the classical approximation the spins are vectors which form the spiral structure with a pitch angle $\varphi$ between neighboring spins and canted angle $\theta$

$$
\begin{aligned}
S_{n}^{x} & =\frac{1}{2} \cos (\varphi n) \sin \theta \\
S_{n}^{y} & =\frac{1}{2} \sin (\varphi n) \sin \theta \\
S_{n}^{z} & =\frac{1}{2} \cos \theta
\end{aligned}
$$

The classical energy per site is

$$
\frac{E_{\mathrm{cl}}(\varphi, \theta)}{N}=\frac{1-\Delta}{4}+\frac{\sin ^{2} \theta}{4}[\Delta-\cos \varphi-J(1-\cos (2 \varphi))]
$$

The minimization of the energy (4) over the angles $\varphi$ and $\theta$ shows that there are three regions in $(J, \Delta)$ having different classical energies. In the region I $\left(J<\frac{1}{4}, \Delta<1\right)$ the energy is minimized by the choice of the angles $\varphi=0$ and $\theta=\frac{\pi}{2}$. These angles correspond to the spin configuration with all spins pointing along the $x$-axis and the energy is

$$
E_{\mathrm{cl}, \mathrm{xy}}=0
$$

In the region II $\left(J<\frac{1}{4}, \Delta>1\right)$ and $\left(J>\frac{1}{4}, \Delta-1>\frac{2}{J}\left(J-\frac{1}{4}\right)^{2}\right)$ the minimum of the energy is given by the angle $\theta=0$ (and arbitrary $\varphi$ ). This is the fully polarized state with all spins up (or down) and the energy

$$
E_{\mathrm{cl}, \mathrm{z}}=-N \frac{\Delta-1}{4}
$$

In the region III $\left(J>\frac{1}{4}, \Delta-1<\frac{2}{J}\left(J-\frac{1}{4}\right)^{2}\right)$ the classical approximation shows helical spin structure in the $x-y$ plane. The corresponding angles are

$$
\begin{aligned}
\varphi & =\cos ^{-1} \frac{1}{4 J} \\
\theta & =\frac{\pi}{2}
\end{aligned}
$$

and the classical ground state energy is

$$
E_{\mathrm{cl}, \mathrm{sp}}=-\frac{N}{2 J}\left(J-\frac{1}{4}\right)^{2}
$$

The phase boundaries in the classical approximation for the model (2) are shown in Fig.1 by thin dashed lines. One can see from Fig.1 that the transition between phases I and II takes place on the isotropic line $\Delta=1$. This 


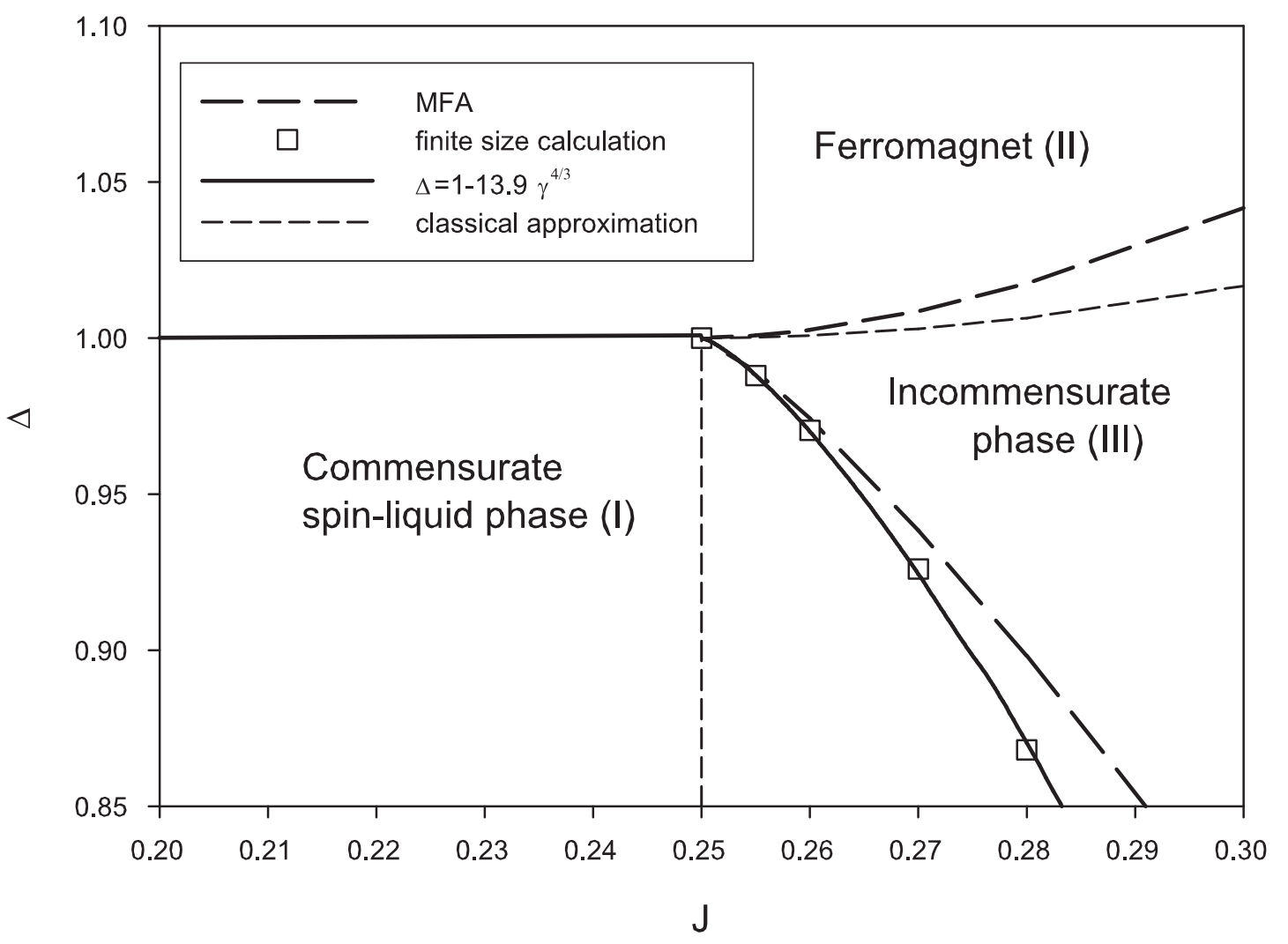

FIG. 1: The phase diagram of the model (2).

transition is a simple spin-flop, which is certainly of the first-order type. In the easy-axis case $\Delta>1$ the increase of the NNN exchange $J$ leads to the first-order transition to the helical phase III with finite value of $\varphi$ (7), which is $\varphi=(8(\Delta-1))^{1 / 4}$ at $\Delta \rightarrow 1$. In contrast to the easy-axis case, in the easy-plane part of the phase diagram the transition to the helical phase occurs at $J=1 / 4$, where the pitch angle $\varphi=0$, indicating the second-order type of this transition.

\section{MEAN FIELD APPROACH}

To study the model (2) we use the variational mean-field approach (MFA) developed in Ref. 21, 29]. According to this approach, we follow the classical picture and transform the local axes on $n$-th site by a rotation about the $Z$ axis by $\varphi n$ and then by a rotation about the $Y$ axis by $\theta$. The transformation to new spin- $\frac{1}{2}$ operators $\eta_{n}$ has a form

$$
\mathbf{S}_{n}=R_{z}(\varphi n) R_{y}(\theta) \eta_{n}
$$

where $R_{y}(\theta)$ and $R_{z}(\varphi n)$ are the operators of the corresponding rotations.

The second step is the Jordan-Wigner transformation to the obtained Hamiltonian in terms of the $\eta$ operators. This transformation maps the $\eta$-spin model onto the model of interacting spinless fermions, which is then treated by the mean-field approximation including superconductor like correlations. The pitch and canted angles $\varphi$ and $\theta$ are variational parameters in this approach. We omit here the details of this approach, because it is simple modification of that done in Ref. 21, 29], and we present here only the results of this approximation.

Generally, the phase diagram of the model (2) in the MFA contains the same three phases as predicted by the classical approximation and the boundaries between the phases are shown in Fig.1 by thick dashed lines. In the region I (see Fig.1) the MFA shows the non-zero magnetization in the easy $x-y$ plane. In the region II the fully polarized state $|\uparrow \uparrow \ldots \uparrow\rangle$ represents the ground state. In the region III the MFA shows helical spin structure in the 
$x-y$ plane. However, as can be seen in Fig.1, the boundary between the phases I and III is substantially shifted. In the MFA this boundary in the vicinity of the point $\left(J=\frac{1}{4}, \Delta=1\right)$ is approximately given by

$$
\alpha \approx 8.05 \gamma^{1.25}
$$

where $\alpha=1-\Delta$ and $\gamma=J-\frac{1}{4}$.

The boundary between the phases II and III in the MFA is described by the equation

$$
\Delta \approx 1+6.3 \gamma^{1.7}
$$

Certainly, there is no LRO in the $x-y$ plane in the phases I and III and in this respect the MFA is incorrect. However, the MFA gives a good estimate for the ground state energy in those phases. For example, in the phase I at $J=0$ the MFA reproduces correctly non-trivial critical exponent for the ground state energy

$$
\delta E_{0} \approx-0.063 N \alpha^{3 / 2}
$$

This estimate differs on $16 \%$ in numerical factor from the exact result [30]

$$
\delta E_{0}=-\frac{N \alpha^{3 / 2}}{3 \sqrt{2} \pi}
$$

The MFA shows that the critical exponent $3 / 2$ for the ground state energy remains up to the point $J=1 / 4$, where the behavior of the ground state energy is changed to

$$
\delta E_{0} \approx-0.07 N \alpha^{9 / 7}
$$

As was shown in Ref. 21] the MFA gives also a good estimate for a critical exponent of the ground state energy in the isotropic case $\Delta=1$ of the helical phase III:

$$
\delta E_{0} \approx-1.585 N \gamma^{12 / 7}
$$

As will be shown below, the estimates of the ground state energies and the phase boundaries in the MFA given by Eqs.(10),(11) are in a good accordance with scaling estimates and finite-size calculations.

\section{PERTURBATION THEORY FOR EASY-PLANE CASE AT $J<1 / 4$}

We are interested in the behavior of the model (2) in the vicinity of the isotropic case $\Delta=1$. For this aim it is natural to develop the perturbation theory (PT) in small parameter $\alpha=1-\Delta$

$$
\begin{aligned}
H & =H_{0}+V_{J}+V_{\delta} \\
H_{0} & =-\sum\left(\mathbf{S}_{n} \cdot \mathbf{S}_{n+1}-\frac{1}{4}\right) \\
V_{J} & =J \sum\left(\mathbf{S}_{n} \cdot \mathbf{S}_{n+2}-\frac{1}{4}\right) \\
V_{\alpha} & =\alpha \sum S_{n}^{z} S_{n+1}^{z}
\end{aligned}
$$

At first let us consider the most simple case $J=0$, where the ground-state energy at $\alpha \ll 1$ is given by Eq. (13). The ground state of $H_{0}$ is ferromagnetic and is degenerated with respect to total $S^{z}$. The perturbation $V_{\alpha}$ splits this degeneracy and in the first order in $\alpha$ we have

$$
\left\langle\psi\left(S^{z}\right)\left|V_{\alpha}\right| \psi\left(S^{z}\right)\right\rangle=\frac{\alpha}{4} \frac{\left(4 S^{z}-N\right)}{N-1}
$$

Thus, the first order shows that one should develop the PT for the lowest state $\left|\psi_{0}\right\rangle$ having total spin $S=\frac{N}{2}$ and $S^{z}=0$. The perturbation series for the ground state energy can be written in a form:

$$
E_{0}(\alpha)=\left\langle\psi_{0}\left|V_{\alpha}+V_{\alpha} \frac{1}{E_{0}-H_{0}} V_{\alpha}+\ldots\right| \psi_{0}\right\rangle
$$


Suppose that the main contributions to the energy are given by low-lying excitations, which for isotropic ferromagnet with the spectrum $\varepsilon_{k}=k^{2} / 2$ behave as

$$
E_{k}-E_{0} \sim N^{-2}
$$

The higher orders of the perturbation series contain more dangerous denominators and, therefore, possibly have higher powers of the infrared divergency. Therefore, we use scaling arguments to estimate the critical exponent for the ground-state energy. Below we will take care only of powers of divergencies and omit numerical factors.

Let us assume that the matrix elements of the perturbation operator $V_{\alpha}$ between low-lying states $\left|\psi_{n}\right\rangle$ involved into the PT (having $S^{z}=0$ but different total $S$ ) at $N \rightarrow \infty$ behave as

$$
\left\langle\psi_{i}\left|V_{\alpha}\right| \psi_{j}\right\rangle \sim \alpha
$$

Collecting the most divergent parts in all orders of the PT, the correction to the ground state energy takes a form:

$$
E_{0}(\alpha)=\left\langle\psi_{0}\left|V_{\alpha}\right| \psi_{0}\right\rangle \sum_{m=0}^{\infty} c_{m} x^{m}=\alpha f_{\alpha}(x)
$$

where $c_{m}$ are unknown constants and

$$
x \sim \frac{\left\langle\psi_{i}\left|V_{\alpha}\right| \psi_{k}\right\rangle}{E_{k}-E_{0}} \sim \alpha N^{2}
$$

is a scaling parameter, which absorbs the infrared divergencies.

The scaling function $f_{\alpha}(x)$ at $x \rightarrow 0$ is given by the first order correction. In the thermodynamic limit $(x \rightarrow \infty)$ the behavior of $f_{\alpha}(x)$ is generally unknown, but the natural condition $E_{0}(\alpha) \sim N$ at $N \rightarrow \infty$ requires

$$
f_{\alpha}(x) \sim \sqrt{x}
$$

and, finally

$$
E_{0}(\alpha) \sim-N \alpha^{3 / 2}
$$

The obtained expression is in agreement with the exact equation (13) for the ground state energy, which justifies our assumption about the behavior of the matrix elements (20).

Moreover, exploiting the fact that the system in the region $\Delta<1$ is in a spin-liquid phase, the correction to the ground-state energy has a form [31]

$$
E_{0}=N e_{0}-\frac{\pi c v_{\text {sound }}}{6 N}
$$

where $e_{0}$ is the ground state energy at $N \rightarrow \infty$ and the central charge is $c=1$ in our case.

In order to reproduce such $1 / N$ correction to the energy, the asymptotic of $f_{\alpha}(x)$ at large $x$ should have a form

$$
f_{\alpha}(x)=a \sqrt{x}+\frac{b}{\sqrt{x}}
$$

with some constants $a$ and $b$.

So, from Eq.(21) we find

$$
E_{0}(\alpha)=-N a \alpha^{3 / 2}-\frac{b \alpha^{1 / 2}}{N}
$$

and, therefore,

$$
v_{\text {sound }} \sim \sqrt{\alpha}
$$

which agrees with an exact result $v_{\text {sound }}=\sqrt{\alpha / 2}$ at $\alpha \ll 1$ [32]. Thus, the scaling estimates give us the correct exponent for the sound velocity as well.

Now let us consider the PT (16) containing both channels $V_{\alpha}$ and $V_{J}$. In order to estimate the powers of divergency of high-orders in this PT one needs to know the $N$-dependence of the matrix elements $\left\langle\psi_{i}\left|V_{J}\right| \psi_{j}\right\rangle$. In general, it is 
unknown. However, one can restore these matrix elements from the known exact expression for NNN spin correlator in the ground state $\left|\psi_{0}(\alpha)\right\rangle$ at $J=0$ and some small value of $\alpha$ [33]:

$$
\left\langle\psi_{0}(\alpha)\left|\left(\mathbf{S}_{n} \cdot \mathbf{S}_{n+2}-\frac{1}{4}\right)\right| \psi_{0}(\alpha)\right\rangle=-\frac{\sqrt{2}}{3 \pi} \alpha^{3 / 2}
$$

or, in other words,

$$
\left\langle\psi_{0}(\alpha)\left|V_{J}\right| \psi_{0}(\alpha)\right\rangle=-\frac{\sqrt{2}}{3 \pi} \alpha^{3 / 2} J N
$$

On the other hand, collecting all contributions of the PT to the linear term in $J$ similar to done in Eq.(21), we arrive at a scaling form in small parameter $\alpha$

$$
\left\langle\psi_{0}(\alpha)\left|V_{J}\right| \psi_{0}(\alpha)\right\rangle \sim\left\langle\psi_{i}\left|V_{J}\right| \psi_{j}\right\rangle \cdot f_{J}(x)
$$

with $x=\alpha N^{2}$.

The comparison of Eqs.(30) and (31) immediately leads to the results

$$
\left\langle\psi_{i}\left|V_{J}\right| \psi_{j}\right\rangle \sim J N^{-2}
$$

and

$$
f_{J}(x) \sim x^{3 / 2}
$$

So, the matrix elements $\left\langle\psi_{i}\left|V_{J}\right| \psi_{j}\right\rangle$ are small enough to eliminate dangerous denominators:

$$
y \sim \frac{\left\langle\psi_{i}\left|V_{J}\right| \psi_{j}\right\rangle}{E_{k}-E_{0}} \sim J
$$

which, in turn, implies the absence of infrared divergencies in $V_{J}$ channel. Thus, the perturbation $V_{J}$ does not form a scaling parameter and the ground state energy has regular expansion in $J$.

It is natural to expect that the behavior of the matrix elements of the type (32) remains the same up to the point $J=1 / 4$. It results in the expression for the ground state energy at $J<1 / 4$ :

$$
E_{0}=-N \alpha^{3 / 2} g_{J}(J)
$$

where $g_{J}(J)$ is some unknown smooth function, which at small $J$ has the expansion in accordance with exact results (13), (30):

$$
E_{0}=-\frac{N \alpha^{3 / 2}}{3 \sqrt{2} \pi}(1+2 J)
$$

However, at approaching to the point $J=1 / 4$ one should take into account that the excitation spectrum is $\varepsilon_{k}=\left(\frac{1}{2}-2 J\right) k^{2}$ and the excitation energies become

$$
E_{k}-E_{0} \sim \frac{\frac{1}{4}-J}{N^{2}}
$$

This modifies the scaling parameter

$$
x \sim \frac{\alpha N^{2}}{\frac{1}{4}-J}
$$

and the expression for the energy

$$
E_{0} \sim-\frac{N \delta^{3 / 2}}{\sqrt{\frac{1}{4}-J}}
$$

Similarly, the sound velocity at $J \rightarrow 1 / 4$ behaves as

$$
v_{\text {sound }} \sim \sqrt{\frac{1}{4}-J} \sqrt{\alpha}
$$




\section{PERTURBATION THEORY NEAR THE TRANSITION POINT $J=1 / 4, \Delta=1$}

At $J=1 / 4$ and $\Delta=1$ the ferromagnetic ground state becomes degenerated with a singlet spiral state [25]. At $\Delta<1$ the ground state obviously lies in the $S^{z}=0$ sector. Therefore, in order to determine the transition line between the phases I and III one should develop the PT both to the ferromagnetic state with $S^{z}=0$ and to the singlet spiral state.

\section{A. The PT to the ferromagnetic state with $S^{z}=0$}

Let us represent the Hamiltonian in a form

$$
\begin{aligned}
H & =H_{0}+V_{\delta}+V_{\gamma} \\
H_{0} & =-\sum\left(\mathbf{S}_{n} \cdot \mathbf{S}_{n+1}-\frac{1}{4}\right)+\frac{1}{4} \sum\left(\mathbf{S}_{n} \cdot \mathbf{S}_{n+2}-\frac{1}{4}\right) \\
V_{\alpha} & =\alpha \sum S_{n}^{z} S_{n+1}^{z} \\
V_{\gamma} & =\gamma \sum\left(\mathbf{S}_{n} \cdot \mathbf{S}_{n+2}-\frac{1}{4}\right)
\end{aligned}
$$

We assume that the behavior of the matrix elements of operators $V_{\alpha}$ and $V_{\gamma}$ remains the same as in the region $J<1 / 4$ (see Eq.(20) and Eq.(32)). However, the scaling parameters are modified due to the changing in one-particle excitation spectrum, which is $\varepsilon_{k}=k^{4} / 8$ at $J=1 / 4$. So, the low-lying excited states involved in the PT (41) behave as

$$
E_{k}-E_{0} \sim N^{-4}
$$

Now according to Eqs.(22), (34) both channels $V_{\alpha}$ and $V_{\gamma}$ produce the scaling parameters:

$$
\begin{aligned}
& x=\alpha N^{4} \\
& y=\gamma N^{2}
\end{aligned}
$$

Thus, as follows from Eq.(21) exactly at $J=1 / 4(y=0)$ the ground state energy can be written in a scaling form

$$
E_{0}(\alpha)=-N \alpha^{5 / 4} f(x)
$$

This scaling and the critical exponent is confirmed by numerical calculations, where the function $f(x)$ is calculated on finite chains with different $N$ and $\alpha$ for the ground state with $k=0$ (see Fig.2). As one can see on Fig.2, all data lie perfectly on one curve $f(x)$ and in the thermodynamic limit the function $f(x) \rightarrow 0.08$. We show in Fig.2 that the same scaling (44) is valid for the lowest excited state with $k=\pi$ as well and that the corresponding scaling function has the same thermodynamic limit $f(x) \rightarrow 0.08$.

The system at $J=1 / 4$ and $\alpha>0$ is in a spin-liquid phase, which is verified by $1 / N$ behavior of low-lying excitations calculated on finite chains (see Fig.3). From the scaling equation (44) we can extract also the critical exponent for the sound velocity:

$$
v_{\text {sound }} \sim \alpha^{3 / 4}
$$

In case when both $V_{\alpha}$ and $V_{\gamma}$ play simultaneously, the scaling estimates (43) give

$$
E_{0}(\alpha, \gamma)=-N \alpha^{5 / 4} f(x, y)
$$

In the thermodynamic limit, when both $x \rightarrow \infty$ and $y \rightarrow \infty$, the scaling function $f(x, y)$ becomes a function of one variable (independent on $N$ )

$$
\nu=\frac{y^{2}}{x}=\frac{\gamma^{2}}{\alpha}
$$

and the ground state energy takes a form

$$
E_{0}(\alpha, \gamma)=-N \alpha^{5 / 4} g(\nu)
$$




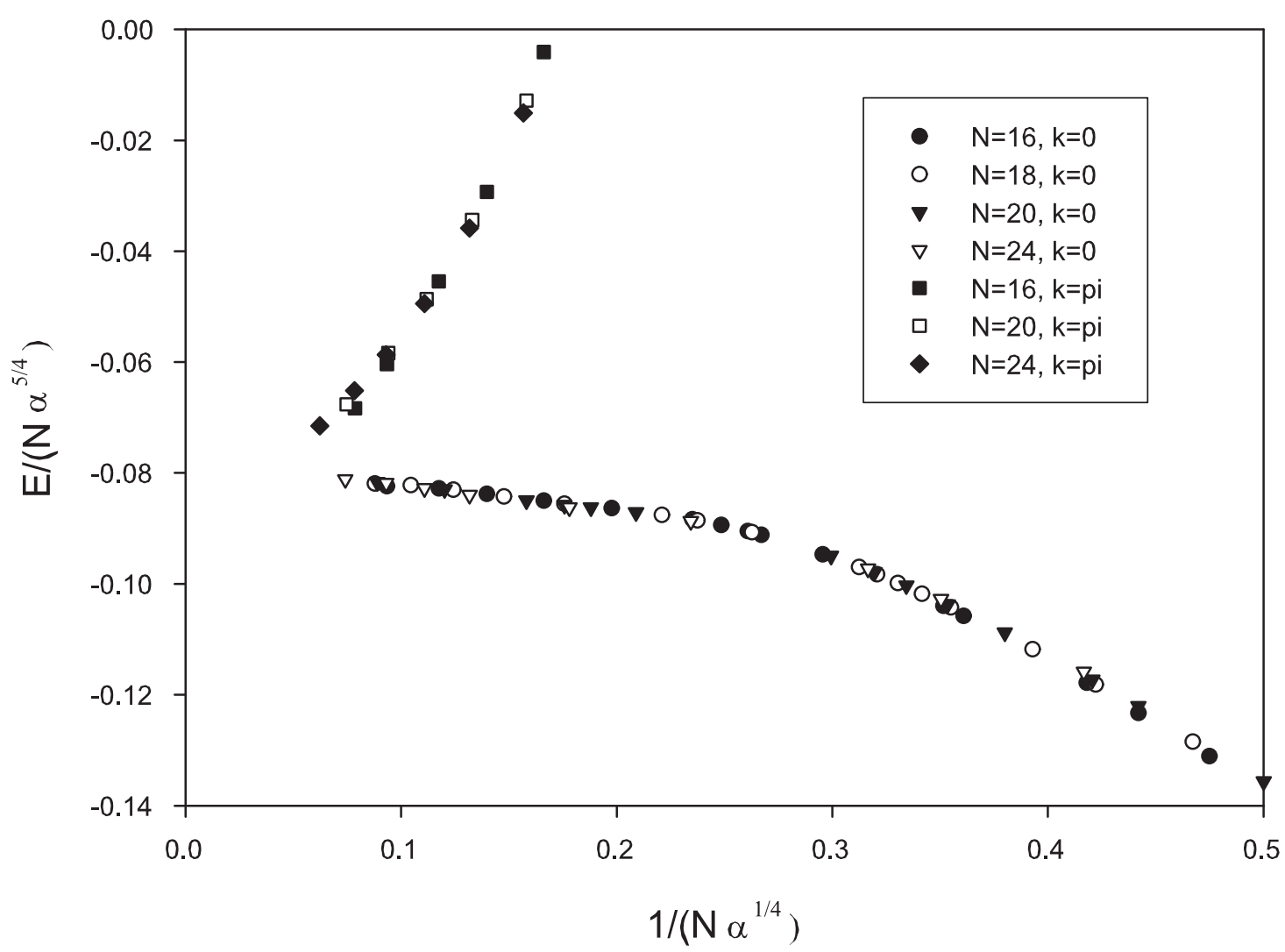

FIG. 2: The scaling function $f(x)$ in Eq. (444) for the ground state energy and the lowest excited state at $J=1 / 4$.

\section{B. The PT to the singlet spiral state}

The PT to the singlet spiral state with pitch angle $\varphi$ in the isotropic case $\alpha=0$ was developed in Ref. 34], where it was found that the energy has a scaling form

$$
E(0, \gamma, \varphi)=-N \frac{\gamma \varphi^{2}}{2}+N \varphi^{5} f\left(\gamma N^{2}, \varphi N\right)
$$

where the first term comes from the first order of the PT in $\gamma$ and the second one originates from the scaling estimates of the infrared divergencies of higher-orders of the PT.

Comparison of Eq.(46) and Eq.(49) leads to a general expression for the energy, which correctly reduces to both cases at $\varphi \rightarrow 0$ and $\alpha \rightarrow 0$

$$
E(\alpha, \gamma, \varphi)=-N \frac{\gamma \varphi^{2}}{2}+N \varphi^{5} f\left(\alpha N^{4}, \gamma N^{2}, \varphi N\right)
$$

(in fact, this equation can be derived in a similar manner as was done in Ref. [34])

In the thermodynamic limit, when all variables in the scaling function in Eq.(50) tends to infinity, the scaling dependence transforms to a function of two variables

$$
E(\alpha, \gamma, \varphi)=-N \frac{\gamma \varphi^{2}}{2}+N \varphi^{5} g(\mu, \nu)
$$

where

$$
\begin{aligned}
\mu & =\frac{\alpha}{\varphi^{4}} \\
\nu & =\frac{\gamma^{2}}{\alpha}
\end{aligned}
$$




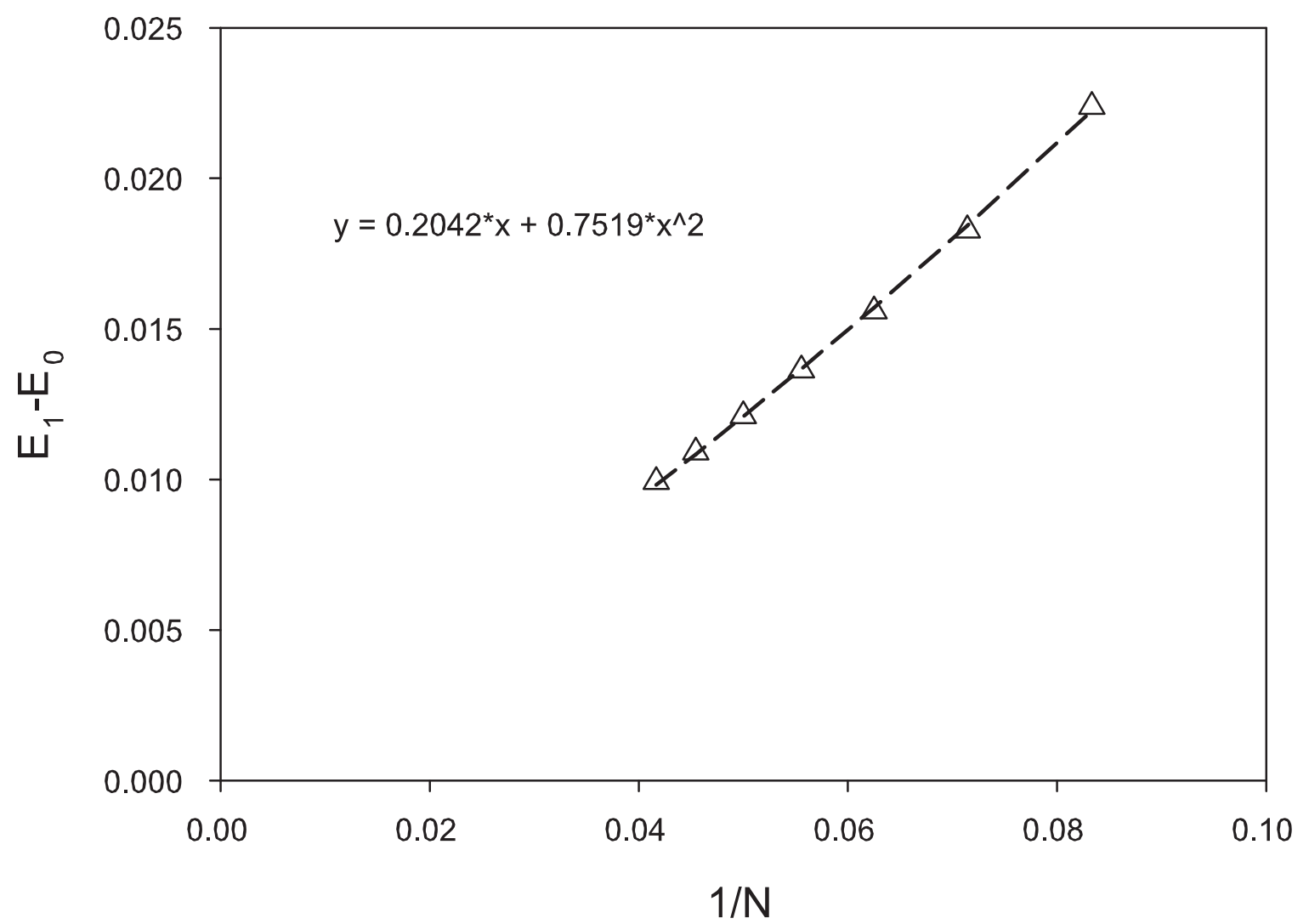

FIG. 3: $N$-dependence of the energy gap between the ground state and the lowest excitation of the model (2) at $J=1 / 4$ and $\Delta=0.96$.

Generally, the function $g(\mu, \nu)$ is unknown. However, we can identify some of its properties. At first, in the limit $\varphi \rightarrow 0$ we should reproduce Eq.(48). Moreover, in the spin-liquid phase the spiral states with $\varphi \sim N^{-1}$ should describe sound-like excitations with the sound velocity (45). These requirements suggest that in the limit $\mu \rightarrow \infty$ the function $g(\mu, \nu)$ has an asymptotic behavior

$$
\lim _{\mu \rightarrow \infty} g(\mu, \nu) \sim-\mu^{5 / 4} g_{1}(\nu)+\mu^{3 / 4} g_{2}(\nu)+o\left(\mu^{3 / 4}\right)
$$

One can check, that this expression reproduces the sound-like excitations at $\gamma=0$ :

$$
E(\alpha, 0, \varphi)-E(\alpha, 0,0) \sim N \alpha^{3 / 4} \varphi^{2} \sim \frac{\alpha^{3 / 4}}{N}
$$

In the limit $\mu \rightarrow 0$, according to Ref. [34] we have

$$
\lim _{\mu \rightarrow 0} g(\mu, \nu) \sim A+o(1)
$$

where constant $A$ describes the excitation spectrum at the transition point $\alpha=0$ and $\gamma=0$. Finite-size calculations give for this constant the value $A \approx 0.0065$.

Summarizing all above we extract explicitly the corresponding terms and obtain the following expression:

$$
\frac{1}{N} E(\alpha, \gamma, \varphi)=-\frac{\gamma \varphi^{2}}{2}-\alpha^{5 / 4} g_{1}(\nu)+\alpha^{3 / 4} \varphi^{2} g_{2}(\nu)+A \varphi^{5}+\varphi^{5} g_{3}(\mu, \nu)
$$

where the function $g_{3}(\mu, \nu)$ has limits

$$
\begin{aligned}
\lim _{\mu \rightarrow 0} g_{3}(\mu, \nu) & \sim o(1) \\
\lim _{\mu \rightarrow \infty} g_{3}(\mu, \nu) & \sim o\left(\mu^{3 / 4}\right)
\end{aligned}
$$


The minimization of the energy (56) over the pitch angle $\varphi$ gives equation for $\varphi_{\min }$

$$
\frac{\partial E(\alpha, \gamma, \varphi)}{\partial \varphi}=0
$$

or after some algebra

$$
\gamma=\varphi^{3}\left[5 A+2 \mu^{3 / 4} g_{2}(\nu)+5 g_{3}(\mu, \nu)-4 \mu \frac{\partial g_{3}(\mu, \nu)}{\partial \mu}\right]
$$

We see that in the isotropic limit $\alpha \ll \varphi^{4}(\mu \rightarrow 0)$ the pitch angle is defined by the constant term in right-hand side of Eq.(59) (using Eq.(57))

$$
\varphi_{\min }=\left(\frac{\gamma}{5 A}\right)^{1 / 3}
$$

which reproduces the result of Ref. 34].

In order to find the commensurate-incommensurate transition line, where the pitch angle $\varphi_{\min }$ vanish, it is more convenient to rewrite Eq.(59) in a form:

$$
\frac{\gamma}{\alpha^{3 / 4}}-2 g_{2}(\nu)=\frac{1}{\mu^{3 / 4}}\left[5 A+5 g_{3}(\mu, \nu)-4 \mu \frac{\partial g_{3}(\mu, \nu)}{\partial \mu}\right]
$$

From Eq.(61) and Eq.(57) one can see that the right-hand side of Eq.(61) tends to zero at $\mu \rightarrow \infty$, which corresponds to the limit $\varphi \rightarrow 0$. The left-hand side of (61) is independent on $\varphi$ and vanishes on the transition line:

$$
\gamma=2 g_{2}(0) \alpha^{3 / 4}
$$

(we note, that on the transition line (62) $\nu=0$ ).

Hence, at approaching to the transition line (62) the pitch angle $\varphi_{\min }$ smoothly goes to zero. So, the line (62) determines the second-order transition line between the commensurate spin-liquid phase I with $\varphi=0$ and the incommensurate spiral phase III with $\varphi \neq 0$.

Another question that can be studied concerns the low-lying excitations in the incommensurate phase. According to Eq.(58) the behavior of the energy near $\varphi_{\min }$ is expanded as:

$$
E(\alpha, \gamma, \varphi)=E\left(\alpha, \gamma, \varphi_{\min }\right)+\frac{\left(\varphi-\varphi_{\min }\right)^{2}}{2} \frac{\partial^{2} E(\alpha, \gamma, \varphi)}{\partial \varphi^{2}}
$$

The second-order derivative of the energy at $\varphi=\varphi_{\min }$ can be estimated as

$$
\frac{\partial^{2} E\left(\alpha, \gamma, \varphi_{\min }\right)}{\partial \varphi^{2}} \sim N \gamma
$$

Thus, the states with

$$
\varphi_{k}=\varphi_{\min } \pm \frac{2 \pi}{N} k
$$

describe gapless excitations with the energy

$$
\delta E \sim \frac{\gamma}{N}
$$

Certainly, there is no helical LRO in the spiral phase and the spin correlations decay on large distances. However, the nature of the spiral phase manifest itself in the incommensurate position $q_{\max }$ of the maximum of structure factor

$$
S(q)=\sum_{n, r} e^{i q r}\left\langle\mathbf{S}_{n} \cdot \mathbf{S}_{n+r}\right\rangle
$$

When the $\mathrm{O}(3)$ rotation symmetry is broken by the anisotropic term $V_{\alpha}$, the incommensurate nature of the spiral phase remains in the $x-y$ plane. So, in this case we associate the pitch angle of the spiral $\varphi$ with the position of maximum of the structure factor $q_{\max }$ in the easy plane

$$
S^{x x}(q)=\sum_{n, r} e^{i q r}\left\langle S_{n}^{x} S_{n+r}^{x}+S_{n}^{y} S_{n+r}^{y}\right\rangle
$$


The numerical calculations on finite chains show that for a fixed small value of $\gamma, q_{\max }$ decreases via consecutive sharp jumps on the value $\frac{2 \pi}{N}$ from some finite value at $\alpha=0$ to zero on the transition line

$$
\alpha \approx 13.9 \gamma^{4 / 3}
$$

where the incommensurate phase III terminates (see Fig.1) and the transition into commensurate spin-liquid phase takes place. Thus, the numerical calculation confirms the found critical exponent for the transition line (62). The fact that the pitch angle $\varphi$ tends to zero at approaching to the transition line ensures that this line is the second-order transition.

\section{THE EASY-AXIS CASE}

In the easy axis case for $J<1 / 4$, the fully polarized state $|\uparrow \uparrow \ldots \uparrow\rangle$ is evidently the ground state. In the region $J>1 / 4$ one should compare the fully polarized state energy with the energy of the spiral state. The finite-size numerical calculations show that for a fixed small $\gamma$ the increasing of easy-axis anisotropy leads to the decrease of the pitch angle $\varphi$, but the ground state remains in the sector with total $S^{z}=0$. At some critical value of $\Delta_{c}$ the transition from the state with $S^{z}=0$ and some finite value of the pitch angle $\varphi$ to the fully polarized state occurs. Thus, in contrast to the easy-plane case, the transition from the spiral phase to the ferromagnetic phase is the first-order one.

The finite-size numerical calculations also show that in the spiral region $1<\Delta<\Delta_{c}$, it is sufficient to take into account only the first-order correction in $(\Delta-1)$ to the spiral state. That is the energy of the spiral state is

$$
E_{s p}=-a N \gamma^{5 / 3}-N \frac{\Delta-1}{12}
$$

and the transition to the fully polarized state with the energy

$$
E_{f}=-N \frac{\Delta-1}{4}
$$

takes place at

$$
\Delta_{c}=1+6 a \gamma^{5 / 3}
$$

Unfortunately, the finite-size calculations do not allowed to find the factor $a$ in Eq.(72) because of irregular behavior of $\Delta_{c}$ with $N$. However, we believe that the MFA gives a good estimate for this transition line (11).

\section{SUMMARY}

We have studied spin-1/2 zigzag chain with weakly anisotropic ferromagnetic nearest-neighbor and antiferromagnetic next-nearest-neighbor interactions. It was shown that the ground state phase diagram consists of three phases: the fully polarized ferromagnetic phase, the commensurate spin-liquid phase and the incommensurate phase. Thus, the incommensurate phase established for the isotropic case survives weak anisotropy of interactions, though in this case the incommensurate nature of the ground state reveals itself in the $x-y$ plane.

Using scaling estimates of the infrared divergencies in the perturbation theory we obtained the scaling expression for the ground state energy both for commensurate and incommensurate phases. This allowed us to determine non-trivial critical exponents in the behavior of the phase transition lines, which were confirmed by finite-size calculations. We found also that in the easy-plane case the transition from the commensurate spin-liquid to the incommensurate phase is of the second order one, while in the easy-axis case the transition from the fully polarized state with $S^{z}=S_{\text {max }}^{z}$ to the incommensurate state with $S^{z}=0$ is evidently of the first order.

In this paper we have focused on studying of the model (2), which is a particular case of more general model (1). However, the obtained results for the model (2) remain valid at least qualitatively for the model (1). As an example we present in Fig.4 the phase diagram near the transition point $J=1 / 4$ of the model (1) in the case $\Delta_{1}=\Delta_{2}$. We see that the phase diagram in this case is very similar even quantitatively to that shown in Fig.1. 


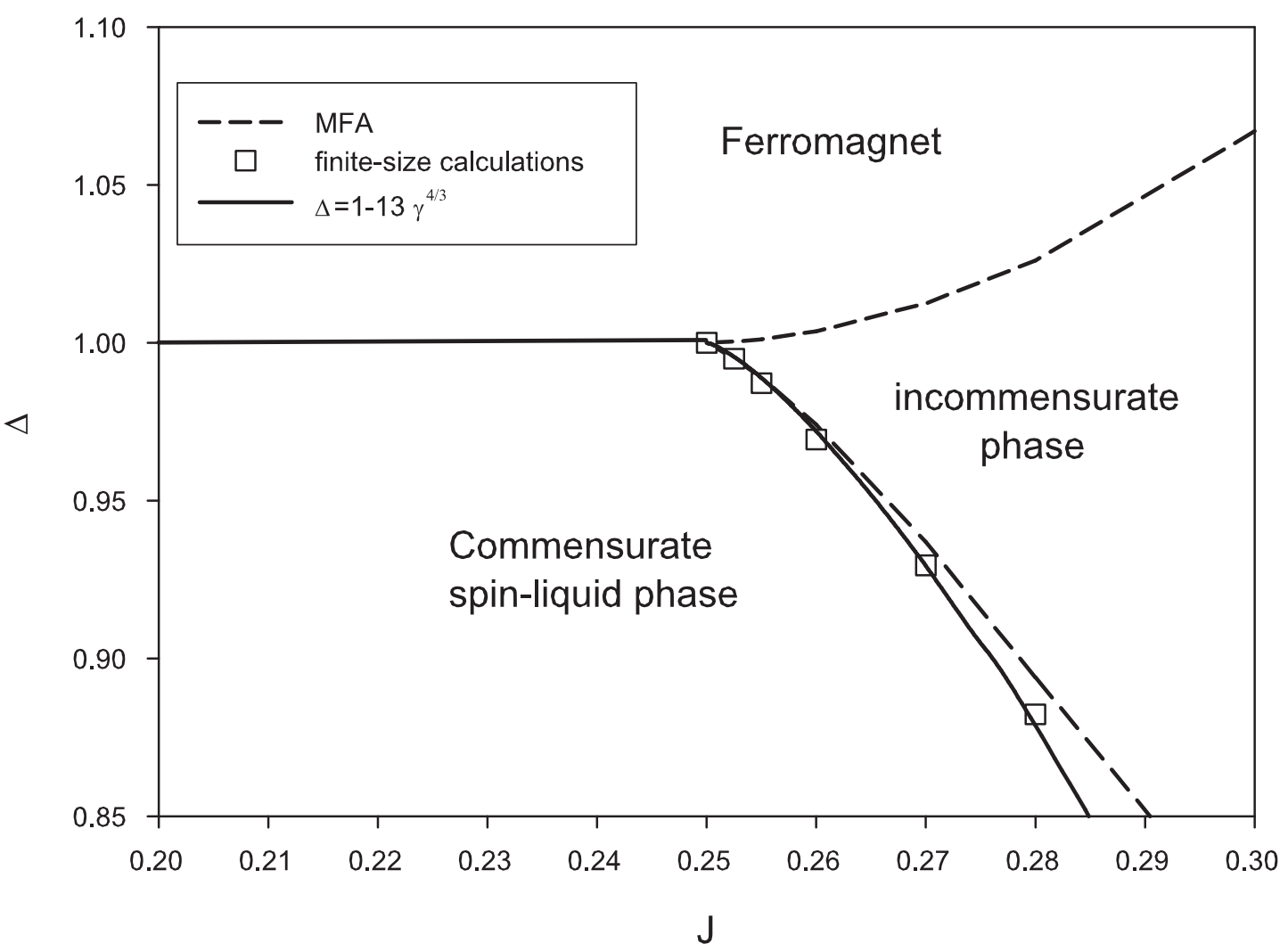

FIG. 4: The phase diagram of the model (1) with $\Delta_{1}=\Delta_{2}=\Delta$.

\section{Acknowledgments}

We would like to thank S.-L.Drechsler and D.Baeriswyl for valuable comments related to this work. D.D. thanks the University of Fribourg for kind hospitality. D.D. was supported by INTAS YS Grant Nr. 05 1094916. The numerical calculations were carried out with use of the ALPS libraries [35].

[1] H.-J. Mikeska and A.K. Kolezhuk, in Quantum Magnetism, Lecture Notes in Physics Vol. 645, edited by U. Schollwöck, J. Richter, D.J.J. Farnell, and R.F. Bishop, Eds. (Springer-Verlag, Berlin, 2004), p. 1.

[2] F.D.M. Haldane, Phys.Rev. B 25, R4925 (1982).

[3] T. Tonegawa and I. Harada, J.Phys.Soc.Jpn 56, 2153 (1987).

[4] K. Nomura and K. Okamoto, Phys.Lett. 169A, 433 (1992).

[5] R. Bursill, G.A. Gehring, D.J.J. Farnell, J.B. Parkinson, T. Xiang, and C. Zeng, J.Phys: Condens.Matter 7, 8605 (1995).

[6] C.K. Majumdar and D.K. Ghosh, J.Math.Phys. 10, 1388 (1969).

[7] S.R. White and I. Affleck, Phys.Rev. B 54, 9862 (1996).

[8] T. Tonegawa and I. Harada, J.Phys.Soc.Jpn 58, 2902 (1989).

[9] A.V. Chubukov, Phys.Rev. B 44, R4693 (1991).

[10] V.Ya. Krivnov and A.A. Ovchinnikov, Phys.Rev. B 53, 6435 (1996).

[11] F. Heidrich-Meisner, A. Honecker, and T. Vekua, Phys.Rev. B 74, 020403(R) (2006).

[12] H.T. Lu, Y.J. Wang, S. Qin, and T. Xiang, Phys.Rev. B 74, 134425 (2006).

[13] A.A. Nersesyan, A.O. Gogolin, and F.H.L. Essler, Phys.Rev.Lett. 81, 910 (1998).

[14] R. Jafari and A. Langari, Phys.Rev. B 76, 014412 (2007).

[15] Y. Mizuno, T. Tohyama, S. Maekawa, T. Osafune, N. Motoyama, H. Eisaki, and S. Uchida, Phys.Rev. B 57, 5326 (1998).

[16] M. Enderle, C. Mukherjee, B. Fak, R.K. Kremer, J.-M. Broto, H. Rosner, S.-L. Drechsler, J. Richter, J. Malek, A. Prokofiev, 
W. Assmus, S. Pujol, J.-L. Raggazzoni, H. Rakoto, M. Rheinstaedter, and H.M. Ronnow, Europhys. Lett. 70, 237 (2005).

[17] S.-L. Drechsler, J. Richter, A.A. Gippius, A. Vasiliev, A.A. Bush, A.S. Moskvin, J. Malek, Y. Prots, W. Schnelle, and H. Rosner, Europhys. Lett. 73, 83 (2006).

[18] M. Hase, H. Kuroe, K. Ozawa, O. Suzuki, H. Kitazawa, G. Kido and T. Sekine, Phys.Rev. B 70, 104426 (2004).

[19] S.-L. Drechsler, J. Richter, R. Kuzian, J. Malek, N. Tristan, B. Buechner, A.S. Moskvin, A.A. Gippius, A. Vasiliev, O. Volkova, A. Prokofiev, H. Rakoto, J.-M. Broto, W. Schnelle, M. Schmitt, A. Ormeci, C. Loison, and H. Rosner, J.Magn.Magn.Mater. 316, 306 (2007).

[20] S.-L. Drechsler, N. Tristan, R. Klingeler, B. Büchner, J. Richter, J. Malek, O. Volkova, A. Vasiliev, M. Schmitt, A. Ormeci, C. Loison, W. Schnelle, and H. Rosner, J.Phys.: Condens. Matter 19, 145230 (2007).

[21] D.V. Dmitriev and V.Ya. Krivnov, Phys.Rev. B 73, 024402 (2006).

[22] D.C. Cabra, A. Honecker and P. Pujol, Eur.Phys.J. B 13, 55 (2000).

[23] C. Itoi and S. Qin, Phys.Rev. B 63, 224423 (2001).

[24] H.P. Bader and R. Schilling, Phys.Rev. B 19, 3556 (1979).

[25] T. Hamada, J. Kane, S. Nakagawa, and Y. Natsume, J.Phys.Soc.Jpn. 57, 1891 (1988); 58, 3869 (1989).

[26] R.D. Somma and A.A. Aligia, Phys.Rev. B 64, 024410 (2001).

[27] H.-A. Krug von Nidda, L.E. Svistov, M.V. Eremin, R.M. Eremina, A. Loidl, V. Kataev, A. Validov, A. Prokofiev and W. Assmus, Phys.Rev. B 65,134445 (2002).

[28] S.-L. Drechsler, O. Volkova, A.N. Vasiliev, N. Tristan, J. Richter, M. Schmitt, H. Rosner, J. Malek, R. Klingeler, A.A. Zvyagin, and B. Buechner, Phys.Rev.Lett. 98, 077202 (2007).

[29] D.V.Dmitriev and V.Ya.Krivnov, Phys.Rev. B 70, 144414 (2004).

[30] C.N. Yang and C.P. Yang, Phys.Rev. 150, 327 (1966).

[31] H.W.J. Bloete, J.L. Cardy and M.P. Nightingale, Phys.Rev.Lett 56, 742 (1986); I. Affleck, Phys.Rev.Lett 56, 746 (1986).

[32] J.D. Johnson, S. Krinsky and B.M. McCoy, Phys.Rev. A8, 2526 (1973).

[33] G.Kato, M.Shiroishi, M.Takahashi, and K.Sakai, J.Phys.A 36, L337 (2003).

[34] D.V. Dmitriev, V.Ya. Krivnov, and J. Richter, Phys.Rev. B 75, 014424 (2007).

[35] F.Alet et al., J.Phys.Soc.Jpn.Suppl. 74, 30 (2005). 